\title{
LA RESPONSABILIDAD SOCIAL COMO ESTRATEGIA DE RELACIONES PÚBLICAS
}

\author{
HAROLD HÜTT HERRERA \\ Escuela de Ciencias de la Comunicación Colectiva, \\ Universidad de Costa Rica \\ gmcostarica@racsa.co.cr
}

\section{RESUMEN}

El presente artículo tiene como objetivo presentar un enfoque descriptivo de los principales lineamientos metodológicos y conceptuales, desde una perspectiva teórica, para el establecimiento de Políticas de Responsabilidad Social en las organizaciones. A partir de lo anterior, se describen los aspectos conceptuales que engloban el término de Responsabilidad Social Empresarial, describiendo sus antecedentes y definiendo sus lineamientos y ámbitos de acción. De manera adicional a los elementos conceptuales, se aportan algunos antecedentes normativos y reglamentarios, tanto del ámbito nacional como internacional, a través de los cuales se ha fortalecido, esquematizado y reconocido este tipo de prácticas en el contexto mundial. Asimismo, hace referencia a varios modelos de gestión, mediante los cuales las organizaciones pueden iniciar un proceso para diseñar e implementar sus políticas y prácticas en esta materia. Finalmente, se concluye con una descripción práctica y una guía básica de cómo pueden ser aplicados modelos de esta naturaleza en diversas organizaciones.

PALABRAS CLAVE: RESPONSABILIDAD SOCIAL, RESPONSABILIDAD SOCIAL EMPRESARIAL, RESPONSABILIDAD CORPORATIVA, MODELOS.

\section{ABSTRACT}

The main objective of this article is to describe, from a theoretical perspective, some concepts and methodologies related to Corporate Social Responsi- bility. This article defines Corporate Social Responsibility, includes historical antecedents, and specifies some guidelines and areas of action. In addition, this article includes several laws and regulations from a local and international perspective. Regulations have strengthened corporate socially responsible practices that have been schematized and recognized worldwide. On the other hand, several management models are described; through these models, the organizations can begin their own process to design and implement their own policies and practices on this matter. Finally, a practical description and a basic guide on how these models can be implemented on different models of the same nature in different organizations are included.

KEYWORDS: SOCIAL RESPONSIBILITY, SOCIAL CORPORATE RESPONSIBILITY, CORPORATE RESPONSIBILITY, MODELS.

\section{INTRODUCCIÓN}

El contexto de la dinámica comercial y productiva del país ha dado un giro importante durante las últimas décadas, a partir de la evolución natural que ha surgido como producto de un Estado cada vez menos interventor y un mercado resultante cada vez más libre, competitivo y marcado por la dinámica global, según se desprende de la información y los datos comparativos que se suministran anualmente en el informe del "Estado de la Nación" (2011). 
De esta forma, las empresas han adquirido cada vez mayor relevancia en materia de generación de riqueza, así como en el aporte que hacen a sus integrantes en términos de calidad de vida, a través de aspectos puntuales tales como: empleo, desarrollo comunal y urbano, impulso del desarrollo sostenible, vigilancia y protección del medio ambiente, entre otros.

Esta dinámica, en algunos casos sugerida, en otros requerida como consecuencia de algunos estándares mundiales, ha impuesto nuevos retos corporativos en materia de "Responsabilidad Social Empresarial" (RSE).

En razón de lo anterior, es el objetivo del presente análisis, consiste en presentar un enfoque descriptivo de los principales lineamientos metodológicos y conceptuales, definidos desde una perspectiva teórica, para el establecimiento de Políticas de Responsabilidad Social en las organizaciones.

Desde esta perspectiva, es necesario tomar en cuenta que el desarrollo de valores éticos, el reconocimiento del factor humano como elemento básico de crecimiento, y consecuentemente la definición de políticas y acciones tendientes a promover una mejor calidad de vida, han confluido en factores positivos como: valores y principios corporativos; respeto a los derechos humanos; relaciones justas, equitativas y transparentes con proveedores; fomento de la lealtad del consumidor y transparencia financiera, entre otros.

Asumir esta responsabilidad no ha sido un reto fácil para las empresas, y sin lugar a dudas, implicó la ruptura de paradigmas y convencionalismos productivos que se constituían como factores antagónicos de este proceso.

Sin embargo, una mayor conciencia social y productiva, ha llevado a los gerentes, propietarios y directivos de las generaciones contemporáneas, a valorar la integralidad como un proceso simbiótico necesario, y a implementar políticas de
Responsabilidad Social, tanto a lo interno como a lo externo de las organizaciones, procurando una mejor calidad de vida para el personal; y lograr así buenos índices productivos, sin perder de vista su compromiso social, económico y ambiental.

Es así como ha venido cambiando la forma de medir el impacto de las empresas, y la concepción acerca de cómo estas inciden en su entorno, en términos socioeconómicos y ambientales.

Lo anterior ha contribuido a definir los lineamientos en materia de responsabilidad social, en razón del rol protagónico que las organizaciones desempeñan en sus respectivas comunidades. De hecho, y según afirman Ogliastri y Flores (2007) indican: "El poder e influencia de las empresas como las conocemos hoy no tiene precedentes" (p.28)

De esta forma, la "responsabilidad social" no es un elemento intangible, sino que por el contrario, es un compromiso corporativo concordante con la filosofía y la política empresarial y productiva, para asumir retos concretos, cumplir estándares y acatar normas, que por convicción y legitimación social, se tornan como elementos obligatorios de mutuo beneficio.

Desde luego, queda intrínsecamente planteada la responsabilidad ineludible que cada uno de nosotros debe asumir como agente de cambio, dentro de cada organización, en cada sector productivo y económico, para fomentar el desarrollo de una mejor sociedad para todos.

Considerar el actuar de una empresa como organización que desempeñe un papel clave en la sociedad, no solamente restringido a la creación de riqueza y empleo, es cada vez más aceptada y los viejos esquemas conceptuales de la escuela van poco a poco perdiendo relevancia(Cordero, 2011, p.4). 


\section{¿Qué es responsabilidad social empresarial (RSE)?}

De acuerdo con Alfaro y Rivera (2010), la RSE:

... es la posibilidad de ir más allá de la filantropía, del cuidado-protección del medio ambiente o del logro de beneficios limitados para la organización en su totalidad. Está supeditada a que las empresas puedan manejar e implementar una concepción integral de la RSE, ya que existe una notable carencia de estrategias corporativas para su implementación y para lograr ventajas competitivas. Este es uno de los desafíos principales que aún persisten en el caso costarricense y en general, en el caso latinoamericano(p. 10).

De este modo, se demuestra la relevancia que ha venido adquiriendo esta materia, lo cual es reforzado por Ogliastri y Flores (2007), quienes señalan que: "Las empresas se han convertido en la fuerza más poderosa en el planeta globalizado, y junto con este poder han crecido también las expectativas de una responsabilidad empresarial de mayor alcance" (p.28).

Como es lógico, las organizaciones han ido madurando este concepto, desde su concepción hasta nuestros días, en razón de lo cual, Ogliastri y Flores nos llevan a hacer un sintético, pero esclarecedor recorrido sobre la evolución de las consideraciones alrededor de la RSE. Señalan en su estudio (2007), que los antecedentes más recientes en la historia acerca del papel de los negocios en la sociedad y su regulación, datan de la segunda revolución industrial, a finales del siglo XIX e inicios del XX. Fue en aquel momento donde el proceder de las empresas empezó a impactar a las comunidades donde estaban inmersas, situación que dio lugar a un debate en Estados Unidos y que se materializó en una serie de leyes que buscaban influir en la conducta empresarial (Ogliastri y Flores, 2007, p.29).
En cambio, otros autores coinciden en que este término no se acuña, sino hasta la década de los setenta, y mencionan algunos otros hechos trascendentales de la primera mitad del siglo $X X$. Estos aseveran que el origen de la RSE tuvo lugar en el período de la depresión económica, alrededor de 1929, ya que esta trajo consigo una serie de eventos que afectaron sensiblemente a la población.

Uno de los hitos más importantes en este proceso, fue el Pacto Global de las Naciones Unidas (UNGC, por sus siglas en inglés), el cual "está conformado por diez principios básicos de adopción voluntaria, que abordan los temas de derechos humanos, estándares laborales, medio ambiente y prácticas anti-corrupción" (Ogliastri y Flores, 2007, p.30).

Estos 10 principios son los siguientes:

1. Las empresas deben apoyar y respetar la protección de los derechos humanos proclamados a nivel internacional.

2. No ser cómplice de abusos de los derechos humanos.

3. Las empresas deben respetar la libertad de asociación y el reconocimiento de los derechos a la negociación colectiva.

4. Las empresas deben apoyar la eliminación de toda forma de trabajo forzoso o realizado bajo coacción.

5. Las empresas deben apoyar la erradicación del trabajo infantil.

6. Las empresas deben apoyar la abolición de las prácticas de discriminación en el empleo y la ocupación.

7. Las empresas deberán apoyar el enfoque preventivo frente a los retos medioambientales. 
8. Las empresas deben fomentar las iniciativas que promuevan una mayor responsabilidad ambiental.

9. Las empresas deben favorecer el desarrollo y la difusión de las tecnologías respetuosas con el medio ambiente.

10. Las empresas deben trabajar contra la corrupción en todas sus formas, incluyendo la extorsión y el soborno.

Este antecedente retoma el auge de la RSE en un contexto más reciente, dado que según Ogliastri y Flores (2007), para el año 2006, un total de 3.700 organizaciones de diversos tipos se habían comprometido a implementar los principios del UNGC.

Aunado a lo anterior, se emite la norma ISO 26000, la cual a diferencia de otros estándares, no intenta ser certificable, sino convertirse en una guía para adoptar prácticas de gestión de RSE, cuya adopción es de carácter voluntario.

Esta norma proporciona orientación sobre los principios que subyacen en la responsabilidad social, su reconocimiento y el involucramiento con las partes interesadas, las materias fundamentales, los asuntos que la constituyen y sobre las formas de integrar un comportamiento socialmente responsable en la organización(INTECO, 2010, p.6).

Por su parte, la Asociación Empresarial para el Desarrollo (AED), ente regulador de la materia en Costa Rica, toma como base la de WBCSD (World Business Council for Sustainable Development), y plantea cómo una definición de este término:

...el compromiso permanente de las empresas para aumentar su competitividad mientras contribuyen activamente al desarrollo sostenible de la sociedad costarricense mediante acciones concretas y medibles dirigidas a solucionar los problemas prioritarios del país. La RSE no es simple filantropía, ni se limita a un programa o proyecto específico, sino que se trata de una nueva forma de gestionar negocios, mediante relaciones responsables con cada uno de los stakeholders o partes interesadas. La RSE debe permear todas las acciones de una empresa, ya que se trata de una estrategia (AED, 2012).

Otras definiciones acuñadas sobre este término, son las siguientes:

Responsabilidad Social Empresarial es el continuo compromiso de los negocios para conducirse éticamente y contribuir al desarrollo económico mientras mejoran la calidad de vida de sus empleados y familias, así como de la comunidad local y sociedad en general (Holme y Watts, citados por Oligastri y Flores 2009, p.2).

Responsabilidad Social es la forma de conducir los negocios de una empresa de tal modo que ésta se convierta en corresponsable por el desenvolvimiento social. Una empresa socialmente responsable es la que posee la capacidad de escuchar los intereses de las diferentes partes (accionistas, empleados, prestadores de servicio, proveedores, consumidores, comunidad, Gobierno y medio ambiente) e incorporarlos en el planteamiento de sus actividades, buscando atender las demandas de todos ellos y no únicamente las de los accionistas o propietarios (Instituto Ethos de Brasil, citado por Oligastri y Flores, 2009, p.2).

La Responsabilidad social empresarial es el compromiso de una compañía de operar de manera económica, social y ambientalmente sostenible mientras reconoce los intereses de sus públicos de interés. Los públicos de interés incluyen inversionistas, clientes, empleados, socios de negocios, comunidades 
locales, el ambiente y la sociedad en general) (Canadian Business for Social Responsability, citados por Oligastri y Flores, 2009, p.4).

Estas, entre otras definiciones citadas por los autores, coinciden en que la RSE es una forma de potenciar el posicionamiento de la empresa, sus productos y/o servicios, a través de la adopción de prácticas económicas, sociales y ambientales, que generen un impacto positivo.

Asimismo, queda claro que las mismas deberán ser adecuadamente difundidas, con el fin de impactar a la opinión pública y consolidar así este propósito, en términos de proyección y posicionamiento.

\section{Modelos de responsabilidad social}

La Gestión Ética y Socialmente Responsable es voluntaria. No obstante, requiere un compromiso que demuestre al menos tres elementos esenciales. En primer lugar, su integración en la estrategia y procesos de la organización. En segundo lugar, el impulso del diálogo y el conocimiento de las expectativas de los grupos de interés. Por último, el fomento de la transparencia y la comunicación donde la publicación de la triple cuenta de resultados (económico, social y ambiental) se ha convertido en una práctica común en las grandes empresas (Forética, 2008, p.6).

Esta base tripartita o triple utilidad como también se le llama, es una forma de medición y divulgación de la responsabilidad social empresarial, que cada vez más empresas han adoptado para dar a conocer sus prácticas sociales, ambientales.

Por medio de esta herramienta, una organización puede publicar, no solo las estrategias que ha implementado y alcanzado con un nivel de impacto exitoso, sino que también puede do- cumentar aquellas en las que no han obtenido buenos resultados, sin que esto represente una vulnerabilidad.

De esto se desprende un modelo, que categoriza la RSE en tres etapas donde una empresa va ascendiendo de una a otra, según el nivel de implementación que logre desarrollar.

La primera es aquella en donde "la empresa se limita a cumplir las leyes y regulaciones; en la siguiente etapa, intenta capturar ventajas competitivas por medio de sus iniciativas de RSE y finalmente, el nivel de "sostenibilidad" representa el estado más avanzado" (Pérez y Cordero, 2010).

Por su parte, Mark Epstein, profesor de la Jones Graduate School of Business, en la Universidad de Houston, Texas, propone un modelo base para desarrollar mediciones de impacto de las distintas acciones de RSE sobre las utilidades, integrando así la gestión social-ambiental y los resultados financieros (Pérez y Cordero, 2010).

Los primeros elementos que deben considerarse son los insumos, que se refieren al contexto interno, externo, de negocios en el cual la empresa opera, y además los recursos disponibles, humanos y financieros. A la luz de estas realidades, los gerentes deben analizar la forma de mejorar la sostenibilidad de su empresa; es decir crear los procesos de RSE. Una vez implementados los procesos se generan dos tipos de productos: reacciones de los involucrados y la medición del desempeño de sostenibilidad. Estos últimos impactan directamente sobre los resultados financieros (Pérez y Cordero, 2010, p.40).

Lo importante de este modelo es que propone un reto para las empresas: la creación de indicadores específicos que visibilicen dicho impacto de manera clara, lo que implica un gran esfuerzo. No obstante, una vez planteados, los resultados de la RSE se evidencian fácilmente. 
Por su parte, la Escuela de Negocios INCAE desarrolló un modelo en el 2004 como respuesta a la petición realizada por la Asociación Empresarial para el Desarrollo, la cual les consignó la responsabilidad de diseñar un modelo de RSE. Después de un estudio de nueve meses y un largo proceso de investigación, en el cual participaron unos 250 representantes de todos los sectores de la sociedad definieron la comunidad, los proveedores, medio ambiente, Estado, consumidores, colaboradores, transparencia y valores y sostenibilidad económica, como los stakeholders formadores del Octágono de RSE (se le otorga esta denominación, en razón de su representación gráfica, a partir de los 8 componentes incorporados en el modelo).

Los componentes del octágono son los siguientes:

1. Cumplimiento de leyes

2. Ética y transparencia

3. Desarrollo de capital humano

4. Mitigación de impactos negativos

5. Beneficios a colaboradores

6. Proyección a las familias

7. Proyección a las comunidades

8. Proyección a nivel nacional y regional

A partir de este octágono, la AED, introdujo en Costa Rica un modelo de Responsabilidad Social Empresarial denominado Heptágono de RSE. Este modelo se homologó en Centroamérica, con el fin de crear la actual Red Centroamericana de RSE: IntegraRSE (AED, 2012). Además, se establecieron indicadores comunes para el sector productivo centroamericano, los cuales se plasmaron en la herramienta IndicaRSE, que permite medir el nivel de conducta socialmente responsable que tienen las organizaciones (AED, 2012).

Gómez y Luis-Bassa (2005), señalan que:
... las empresas con visión de futuro deben estar a la vanguardia de nuevas prácticas de gestión que permitan profundizar su relación con la sociedad. Una de ellas es emprender iniciativas que al complementar su estrategia de negocio, genere valor social en las comunidades que atienden. A fin de cuentas, cada comunidad agrupa un conjunto de consumidores (Gómez y Luis-Bassa, 2005, p.117).

De esta forma, las empresas que asumen la responsabilidad social como parte de su estrategia de negocio tienen más posibilidades de acercarse a los consumidores y determinar sus necesidades, mejorar su imagen pública, posicionarse como una organización que se preocupa por su entorno, diferenciarse de la competencia, expandir sus mercados, además de generar motivación dentro de sus colaboradores y mantener una relación positiva con todos sus stakeholders o públicos de interés.

A su vez, las exigencias del mercado internacional, de la competencia y de los propios consumidores, implican innovar cada vez más, ya que los públicos son más exigentes; ya no se preocupan solo por el producto, sino también por el impacto de los procesos, para que estos se lleven a cabo: "La empresa, al acercar la iniciativa social a su estrategia de negocio, no solo cumple con el loable criterio de sostenibilidad, sino que además abre la posibilidad de alcanzar ventajas competitivas" (Gómez y Luis-Bassa, 2005, p.26).

La RSE al ser implementada, en este caso, manifiesta resultados positivos, tanto a mediano como a largo plazo, y representa beneficios para la organización, sus empleados y familias, así como también para la comunidad (Orsi, 2009, p.38).

Según Ogliastri, Flores y otros (2009. P. 95), el beneficio para el negocio que se deriva de las prácticas de RSE, están impulsando a un número cada vez mayor de empresarios consideren este elemento como un factor competitivo de alto valor estratégico. 
En una encuesta de Price Waterhouse Coopers a 140 gerentes generales de compañías multinacionales basadas en Estados Unidos, aplicada en el 2002, el 89\% de estos gerentes respondió que en cinco años el desarrollo sostenible sería más importante para su modelo de negocios que lo que era en ese momento. El 75\% de esos directivos indicaron haber adoptado algunas prácticas de negocio sostenibles, y entre las motivaciones principales para hacerlo figuraban los efectos positivos en la reputación, ventajas competitivas y ahorros en costos (Ogliastri, Flores y otros 2009, p. 95 y 96).
Es decir, la mayoría de las empresas ha reconocido la RSE, como un elemento estratégico para su proyección y posicionamiento, tanto a nivel local como internacional, según su ámbito de acción.

Para estos efectos, Oligastri y Flores (2009), realizaron un análisis esquemático de los diversos niveles en los cuales las organizaciones se pueden ver beneficiadas, a partir de la incorporación de este tipo de políticas, según se detalla en la Figura 1

\section{BENEFICIOS COMPETITIVOS DE LA RSE}
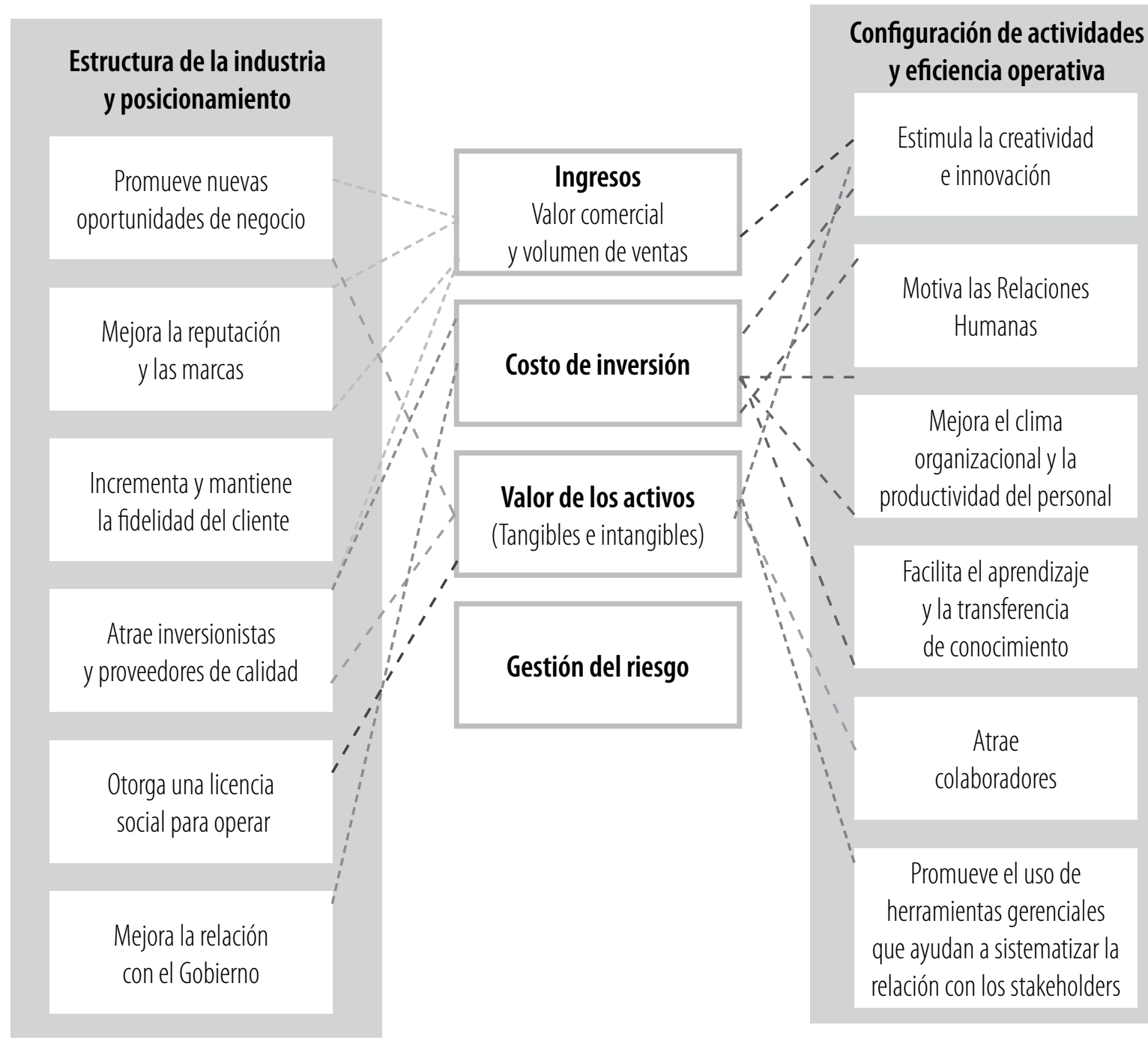

Fuente: Ogliastri, Flores y otros (2009, p. 101). 
Como se deriva de la figura 1, y como ya se mencionó, son múltiples los efectos positivos que pueden derivarse de la implementación de prácticas de RSE, incluyendo la posibilidad de generar nuevas oportunidades de negocio para la organización, o bien, facilitar posibilidades de incursión en ciertos mercados, en los cuales este tipo de prácticas son esenciales para evitar barreras de acceso. Es decir, evitar limitaciones para comercializar en esos mercados, como producto de la exigencia de los mismos, respecto de la adopción de prácticas de RSE por parte de las empresas interesadas en incursionar en su territorio.

\section{¿Cómo puede llevarse a cabo el planteamiento de una estrategia en materia de responsabilidad social empresarial?}

Uno de los primeros aspectos que debe aclararse, es que la filantropía, la caridad, o el apoyo económico -per sé-, a sectores o personas vulnerables, no se constituyen como acciones de Responsabilidad Social. Es decir, este tipo de acciones corresponden a contribuciones o ayudas, que de manera voluntaria, y por iniciativa de los propietarios o grupos dentro de la organización, se llevan a cabo con fines de bien social, pero que no se encuentran enmarcados dentro de una política corporativa de carácter estratégico y permanente.

Para estos efectos se requiere definir cuáles son las áreas o ámbitos de acción, en los cuales, desde la esencia de la organización, se puede generar un aporte social, basado en los principios, lineamientos y la filosofía organizacional, sin dejar de lado su naturaleza básica.

Por ejemplo, y según exponen Escobar, Gutiérrez y otros (2008), Nokia ha convertido la estrategia de responsabilidad social en una parte integral dentro de la toma de decisiones en todos los niveles de sus negocios. Cuenta con varias actividades comunitarias alrededor del mundo, cuyo objetivo son los jóvenes, desde la perspectiva de la educación. Asimismo, desde el año 2000 esta empresa ha invertido más de 25 millones de dólares en programas para el desarrollo de la juventud, que han beneficiado directamente a más de 28.000 jóvenes (p. 32).

Asimismo, en el caso de la empresa Wal-Mart, la RSE es un compromiso corporativo que se refleja en su cultura empresarial, y se fundamenta en sus políticas y en programas concretos de beneficio a la comunidad.

En marzo del 2003, nació la fundación Wal-Mart de México, con el propósito de apoyar programas de desarrollos social sustentables en las comunidades en las que se encuentran sus unidades, y con un enfoque especial en proyectos integrales en nutrición. De esta manera, por medio del trabajo voluntario y brigadas de apoyo con los asociados de todos los niveles, se llevan a cabo programas para contribuir al desarrollo de diferentes comunidades (Escobar, Gutiérrez y otros. 2008. p. 34).

Según Gómez y Luis-Bassa (2005, p.25), es razonable que las empresas procuren obtener beneficios económicos, derivado de sus actividades, dado que es la forma de garantizar una operación sostenible en el tiempo, generar riqueza para sus accionistas, y para sus empleados, a través de la generación de fuentes de empleo. Es así como se fortalece la economía, y se genera una contribución a la sociedad.

Sin embargo, en la actualidad la expectativa es superior a esto, ya que se espera que las empresas implementen actividades específicas de responsabilidad social.

En América Latina, la respuesta al Ilamado a la responsabilidad social que ha emergido -muchas veces circunscrita a "hacer el bien"-no siempre es sostenible. De hecho, diversas empresas venezolanas antecedieron a muchas de las multinacionales en la práctica de auspiciar inversiones de orden social, que a la postre no fueron mantenidas en el tiempo (Gómez y Márquez, citados por Gómez y Luis-Bassa ,2005, p. 25).

Según agregan estos mismos autores, la iniciativa social debe enmarcarse en un marco sostenible y debe generar valor a la empresa. 
De hecho, en muchos casos se genera valor social a partir de la comercialización de sus productos, en razón de que los mismos contribuyen a mejorar la calidad de vida de los consumidores, como es el caso de las empresas farmacéuticas, por ejemplo.

Sin embargo, la expectativa social y un entorno cada vez más competitivo, han implicado que las empresas deban potenciar este valor.

Pocas son las empresas que emprenden una iniciativa social con fines estratégicos. La auspician en espera de mejorar su imagen, lo cual de por sí es una estrategia que, según el caso, favorece a la empresa. Además de imagen, una iniciativa social emprendida con objetivos estratégicos puede traer a la empresa resultados aún más beneficiosos, tales como ampliar el mercado o acercar la empresa a los consumidores. Mediante tal acercamiento la empresa conoce mejor sus necesidades, se percata de cómo el consumidor percibe los productos y servicios que ofrece al mercado y de qué manera podría atenderlo mejor (Gómezy LuisBassa 2005, p. 26).

El principal reto en este proceso, es lograr identificar aquellas actividades y estrategias, que permitan desarrollar iniciativas sociales, las cuales sean parte integral del negocio y cuenten con apoyo, e incluso participación, de accionistas y/o empleados.

Es decir, se trata de implementar actividades que beneficien a la comunidad, al mismo tiempo que generen una ventaja competitiva para la empresa, sea en función de incrementar el consumo de sus bienes o servicios, o de mejorar su posicionamiento y/o liderazgo en el mercado.

Según Gómez y Luis-Bassa (2005, p. 53), existe una gama importante de alternativas para implementar este tipo de prácticas, dentro de las cuales destacan las que se presentan en la figura 2

\section{DIVERSAS OPCIONES PARA ABORDAR UNA INICIATIVA SOCIAL, SEGÚN EXIGENCIAS DE GESTIÓN}

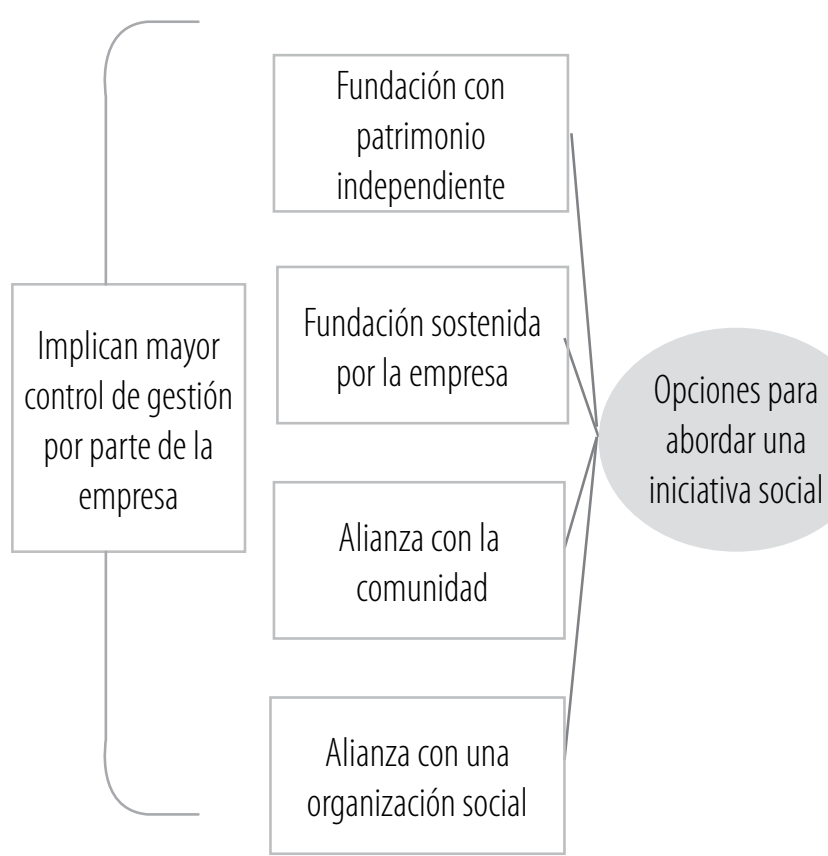

Fuente: Gómez y Luis-Bassa (2005, p. 53)

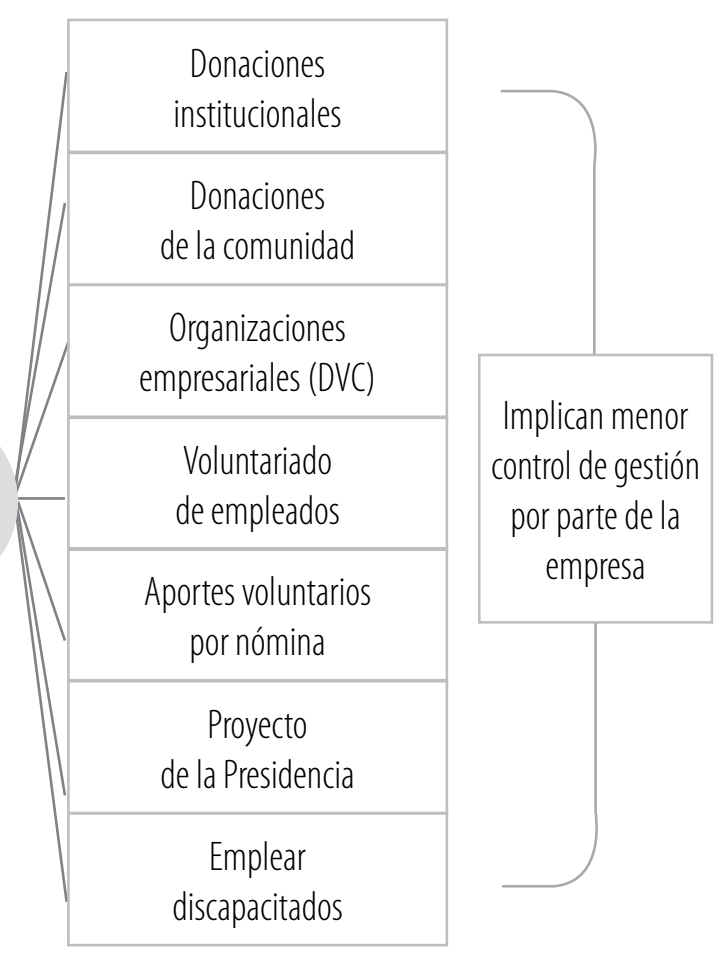


De acuerdo con el esquema anterior, y según detallan Gómez y Luis-Bassa (2005), el hecho de que el control de gestión no necesariamente recaiga sobre la empresa, no implica que las acciones tengan menor efectividad o valor estratégico para la empresa.

Lo importante en esencia, es lograr una adecuada proyección y posicionamiento, a partir de su implementación, partiendo desde luego que dichas acciones tendrán un alto impacto social.

Hay quienes argumentan que la iniciativa social -al igual que la filantropía- no debe vincularse con la estrategia de negocio; consideran que hacerlo tilda a la empresa de oportunista. Por otra parte, el público espera, cada vez más, que las empresas le retribuyan a la sociedad, los beneficios que reciben. La empresa que no divulga su iniciativa social por razones de modestia o con el propósito de mantener un bajo perfil, incumple las expectativas que de ella se tienen. Peor aún, su directiva puede ser acusada de malgastar el patrimonio de los accionistas en actividades que -por no ser debidamente difundidas-, dejan de generar beneficios para la empresa (Gómez y Luis-Bassa 2005, p. 57).

En términos de evaluación, Ogliastri y Flores (2009, p 241-280), establecen una propuesta de indicadores de RSE, los cuales se dividen en las siguientes áreas:

Transparencia, valores

y prácticas anticorrupción

1. Compromisos éticos y prácticas anticorrupción.

2. Relación transparente con los públicos de interés.

3. Gobernanza y control.

4. Disponibilidad de información y reportes de sostenibilidad.

\section{Colaboradores}

1. Gerencia participativa y aprendizaje organizacional.

2. Igualdad de oportunidades y equidad.

3. Capacitación y desarrollo profesional.

4. Uso de mano de obra local.

5. Remuneración, beneficios y jornada laboral.

6. Asociaciones laborales.

7. Manejo de despidos.

8. Salud y seguridad ocupacional.

9. Trabajo forzado, castigos y acoso sexual.

10. Trabajo infantil.

\section{Consumidores}

1. Prácticas de mercadeo y publicidad.

2. Seguridad y calidad de los productos y servicios.

3. Atención al cliente y garantías.

\section{Proveedores}

1. Criterios de selección de proveedores.

2. Apoyo al desarrollo sostenible de proveedores.

\section{Comunidad}

1. Participación en proyectos comunitarios.

2. Trabajo voluntario promovido por la empresa.

3. Reinversión de utilidades y transferencia tecnológica.

4. Patrimonio social. 


\section{Medio ambiente}

1. Compromiso con la protección del medio ambiente y con la educación ambiental.

2. Impacto ambiental de la actividad productiva.

3. Impacto ambiental del producto terminado.

\section{Fortalecimiento de instituciones públicas}

1. Tributación.

2. Participación en proyectos sociales o ambientales con el Gobierno.

3. Transparencia en el papel político de la organización.

4. Gerencia de crisis y riesgo y vulnerabilidad frente a desastres.

Cada uno de los ítems descritos anteriormente es desglosado por los autores en una matriz de puntos específicos, a los cuales debe responderse en dos columnas complementarias, afirmativa o negativamente, y a partir de esto se obtiene una evaluación promedio.

Posteriormente, al final de cada cuadro aparecen cuatro enunciados generales, a manera de conclusión, de los cuales debe ser elegido el que más se ajuste a la realidad de la empresa.

Por ejemplo, en el caso de manejo de crisis y riesgo, al final del formato aparecen los siguientes ítems (Ogliastri, Flores y otros. 2007. p. 280):

a) No cuenta con guías de acción frente a situaciones de crisis y riesgos.

b) Cuenta con un plan de acción frente a situaciones de crisis y riesgos. Sin embargo, este plan no es ampliamente conocido por todos su empleados y raramente se realizan pruebas o simulacros. c) Tienen un plan documentado con guías de acción claras por seguir en situaciones de crisis y riesgo, ampliamente conocido y asimilado por todo el personal. Dicho plan incluye la realización de pruebas y simulacros regulares.

d) En adición a lo anterior, convoca a discusiones periódicas que involucran a empleados, proveedores, clientes, sociedad civil o representantes del Gobierno con la finalidad de discutir el tema e implementar mejoras.

A partir de los descriptivos expuestos se concluye el informe de evaluación y se presentan las conclusiones y recomendaciones respectivas.

\section{Definición y descripción de los niveles en el desarrollo de una estrategia de RSE}

Como parte del objetivo derivado de la presente investigación documental, se ha previsto el desarrollo descriptivo de los principales lineamientos y niveles, bajo los cuales pude ser implementada o planificada la Responsabilidad Social en las organizaciones.

Según Escobar, Gutiérrez y otros (2008, p.10-13), existen varios niveles de implementación de estrategias de RSE, los cuales fueron definidos por la Fundación PROHUMANA:

1. Nivel de estrategia emergente: "corresponden a acciones implementadas por las empresas, las cuales poseen un énfasis tradicional en la relación que se establece con su entorno y con sus trabajadores".

2. Nivel de formalización estratégica: "Es el nivel en donde las compañías detectan la necesidad de organizar y coordinar sus diversas políticas respecto a su vinculación con la comunidad y con sus propios trabajadores en 
busca de generar una sinergía y mejores resultados de dichas acciones. En esta estrategia el proceso de reflexión en torno al tema generalmente coincide con el momento de crear las fundaciones empresariales".

3. Nivel de estrategia formal: "En esta estrategia se da la internalización de la RSE en valores de la compañía, explícitos claramente para cada miembro de ésta, y parte fundamental de sus deberes y derechos".

Asimismo, los autores (Escobar, Gutiérrez y otros. 2008, p.13-17), hacen referencia a varios modelos de aplicación, dentro de los cuales destacan:

1. Modelo Tradicional de Desarrollo Social: "El modelo tradicional de desarrollo social se encuentra cerca del nivel de estrategia emergente. Prima la lógica cristiana de la claridad y la de desarrollo social, por un lado, las nociones éticas valóricas, social y desarrollo e igualitarias, por otro. En este modelo, conviven lógicas tradicionales de relación con la comunidad interna y externa de la empresa, así como visiones en las que predomina un fin de igualdad social y acceso a oportunidades".

2. Modelo Estratégico Solidario: "Este modelo se caracteriza por tener presentes dos componentes fundamentales, la visión estratégica de la RSE, altamente ligada al marketing, denominada por aspectos coyunturales de la lógica estratégica, esto quiere decir que la RSE es vista como una necesidad de mercado en el ámbito de la globalización. El otro componente tiene relación con una visión solidaria de la sociedad, donde la empresa puede aportar a mejorar la condición social de su comunidad adyacente".

3. Modelo estratégico Friedmaniano: "Este modelo posee la característica de estar dominado por una racionalidad económica respecto de la RSE. En este modelo, las acciones que desarrolla la empresa tienen una visión estratégica, fuertemente ligada a la noción friedmaniana, en la que su ámbito de intereses y acción es totalmente auto referido hacia sus trabajadores, accionistas, proveedores y distribuidores, así como a sus consumidores".

Indistintamente del modelo, o del nivel de formalización, lo cierto del caso es que las organizaciones han comprendido que la Responsabilidad Social es un elemento indispensable dentro de su esquema operacional, dado que es la forma de procurar una retribución social, en aras de fortalecer y estimular una relación de dependencia simbiótica, a través de la cual tanto el sector empleador como la comunidad, pueden convivir en un marco de mayor armonía, igualdad y distribución de la riqueza, y desde luego, en un proceso de trabajo conjunto a través del cual se logren producir bienes y servicios competitivos, con altos estándares de calidad y eficiencia, sin desmérito de la calidad de vida y del aporte que de diversas maneras, tanto directas como indirectas, la sociedad facilita el uso y disponibilidad de recursos naturales y humanos.

Es así como se ha logrado generar conciencia en la importancia de que todos hagamos un aporte para mejorar la calidad de vida en cada región, en cada ciudad o país, así como también, impulsemos de manera conjunta el desarrollo, bajo una perspectiva sostenible, transparente, equitativa y socialmente gratificante.

No obstante lo anterior, hay que tomar en cuenta que esta materia es relativamente reciente en nuestro país, por lo que no se cuenta con una trayectoria de implementación que permita definir parámetros o modelos debidamente sustentados, más allá de los lineamientos establecidos en el marco de las experiencias internacionales, y bajo la salvedad del modelo desarrollado por el INCAE, en el cual se perfila una propuesta metodológica para implementar y evaluar la gestión de RSE. 


\section{CONCLUSIONES}

Después de hacer un análisis detallado de los elementos teóricos expuestos anteriormente, se pueden extraer varias conclusiones relevantes.

En primer término, es necesario destacar que la Responsabilidad Social Empresarial (RSE) ha sido reconocida por las organizaciones actuales como una ventaja competitiva, para impulsar el posicionamiento de marca y promover la fidelidad del cliente, entre otros aspectos, lo que ha generado una presión tácita en las organizaciones, para desarrollar e implementar iniciativas de esta naturaleza.

No obstante, es claro que existe una marcada diferencia entre las gestiones de RSE y las acciones de carácter filantrópico. Esta diferencia se genera por el vínculo de estas acciones con la gestión empresarial, y su permanencia en términos de implementación. Es decir, la RSE responde a un esquema estratégico, sostenible y permanente, en tanto las labores filantrópicas son de carácter puntual o esporádico, y no necesariamente el perfil de estas actividades está ligado al ámbito de acción de la empresa.

Sin lugar a dudas, esta gestión ha permitido colaborar de manera significativa al proceso de construcción de la reputación e imagen corporativa, con un alto nivel de aceptación y reconocimiento social, lo que ha permitido una fuerte y acelerada incorporación de políticas de RSE en organizaciones, al margen de sus características particulares.

Aunado a lo anterior, es importante resaltar que el declive que ha experimentado la publicidad, como producto de la saturación mediática y la sobre-exposición de mensajes, ha implicado que las organizaciones deban recurrir a nuevos elementos y posibilidades para llegar a sus usuarios.

Tomando en cuenta los antecedentes y elementos descritos, es claro que la RSE permite generar un alto impacto en la población, si se utiliza de manera adecuada, dado que la gestión social implica un proceso participativo comunitario, a través del cual las personas o públicos meta se ven directamente involucradas, y beneficiadas por los proyectos y actividades que se generan bajo esta perspectiva. Lo anterior, genera un fuerte vínculo entre las partes, así como también, se logra un alto nivel de penetración, en términos de reconocimiento y posicionamiento de marca.

Dentro de las diversas aristas que se pueden resaltar en esta materia, destacan los valores, el cumplimiento de la normativa vigente, la protección al medio ambiente, el impulso de políticas sostenibles y la promoción de relaciones justas, equitativas y transparentes entre patronos y empleados, así como entre empresas y consumidores.

Por su parte, la sociedad ha concebido, de alguna manera, las gestiones de RSE como una forma de lograr un mayor balance económico y social entre las empresas y la comunidad. Esto por cuanto se atribuye a las organizaciones, principalmente aquellas de carácter lucrativo, la obligatoriedad de ser socialmente responsables con su entorno, y desde luego, con sus colaboradores.

A pesar de que estas prácticas son de adopción voluntaria, es evidente que en ciertos mercados su implementación se ha convertido en un requerimiento obligatorio, dado que su adopción está directamente relacionada con barreras comerciales. Para estos efectos se han establecido pautas específicas a través de normas, como es el caso de la ISO 26000.

Es necesario anotar que existen varios modelos y tipos de responsabilidad social, así como parámetros y mecanismos de implementación y evaluación. No obstante, lo que es esencial es que sean concebidos en el marco de política estratégica de la organización, y que sean difundi- 
dos de manera adecuada, a través del uso de herramientas de comunicación idóneas y efectivas.

En síntesis, si la RSE si es implementada de manera adecuada, generará beneficios inmediatos, efectivos y permanentes en diversas áreas tales como: mejoramiento de los índices de productividad, resguardo de la reputación corporativa, posicionamiento, relaciones públicas de la mar$\mathrm{ca}$, relaciones comunitarias, relaciones gubernamentales, y desde luego, relaciones comerciales.

Asimismo y por su parte, implicará mejoras sustanciales en relación con las condiciones laborales que las empresas procurarán para sus empleados, así como en la participación directa y las relaciones permanentes que estas establecerán con sus comunidades inmediatas, e incluso en algunos casos, en el ámbito nacional o internacional.

Este proceso, sin lugar a dudas, obedece a un proceso natural e inherente de cambio en las organizaciones, como producto de su proceso de adaptación al entorno, al mercado, a las nuevas tendencias culturales y a los cambiantes estándares de gestión y productividad, que cada vez más, responden a esquemas integrales, humanistas y solidarios, dejando de lado el esquema productivo de épocas anteriores, el cual sentaba las bases de una sociedad dividida por el acceso a los recursos, y derivado de lo anterior, al establecimiento de claras y marcadas diferencias en el acceso a mejores expectativas en relación con la calidad de vida, el desarrollo personal, económico y social de los diversos estratos y grupos estructurales.

\section{REFERENCIAS}

AED (2012) ¿Qué es RSE? Recuperado de: http://www.aedcr. com/subsection.php?id=36\&section=rse

Alfaro, D. y Rivera, M. (2010). La Responsabilidad Social en empresas nacionales: un acercamiento a la relación gobierno corporativo-colaboradores. Trabajo final de graduación para optar por el grado de licenciatura en Psicología. Universidad de Costa Rica. P. 1, 3, 7, 10.
Consejo Consultivo Nacional de Responsabilidad Social. (s.f.). Lineamientos estratégicos para una agenda de RSE en Costa Rica. Capítulos 3-4.

Cordero, A. (2011). Propuesta de marketing social corporativo para el sector hotelero de la ciudad de Puntarenas, aplicando un análisis de bechmarking competitivo. Trabajo final de graduación para optar por el grado de maestría en Administración de Empresas. Universidad de Costa Rica.

Escobar, R., Gutiérrez, A. y otros (2008). Trabajo de graduación. Modelo de aplicación de Responsabilidad Social Empresarial basado en acciones comunitarias para empresas de la provincia de Guanacaste. Universidad de Costa Rica.

Forética. (2008). SGE 21 Sistema de Gestión Ética y Socialmente Responsable. Documentación curso de gestión de Responsabilidad Social Empresarial.

Gómez, Luis-Bassa (2005) Iniciativa social como estrategia competitiva. Ediciones IESA. P 26, 27, 36, 45, 57, 58, 103, 105, 106, 108, 109, 112, 113, 117, 118.

Instituto de Normas Técnicas de Costa Rica (INTECO). (2010). INTE/ISO 26000:2010.

Programa del Estado de La Nación (2011). Decimoséptimo Informe del Estado de la Nación. San José, Costa Rica. Recuperado de http://www.estadonacion.or.cr

Ogliastri, E. y Flores, J. (2007). INCAE Business School. Concepto y transformación. Acerca de cómo la responsabilidad social evolucionó hasta desaparecer y convertirse en parte de la gerencia estratégica. 1 (2), 28-36.

Ogliastri, E. y Flores, J. y otros (2009). El Octágono. Un modelo para alinear la RSE con la Estrategia. Editorial Norma. INCAE Business School.

Orsi, A. (2009). EKA. La Responsabilidad Social, ¿Un buen Negocio? (296), 38- 40.

Pérez, F. Cordero, A (2010). INCAE Business. Nueva Visión regional en la RSC 1(2) P.34-45

Pérez, F. y Rubio, J. (2007). INCAE Business. ¿Vale la pena ser una empresa responsable? 1 (2), P.42-47.

Recibido: 7 de junio de 2012 Aceptado: 29 junio de 2012 